\title{
ON THE CALCULATION OF THE GENERALISED POISSON FUNCTION
}

\author{
ERkKi Pesonen
}

Helsinki

Drs. H. Bohman and F. Esscher have reported in a recent paper ${ }^{1)}$ an extensive research performed in Sweden on the different methods of calculation of the distribution function of the total amount of claims. In the present paper certain methods are discussed in so far as they are different from those presented in the above quoted paper. The consideration is restricted to the generalised Poisson function even though some results can be easily extended. The author has already commented on some of the results represented in the sequel at a special meeting of the $\mathrm{x} 7$ th International Congress of Actuaries in Edinburgh.

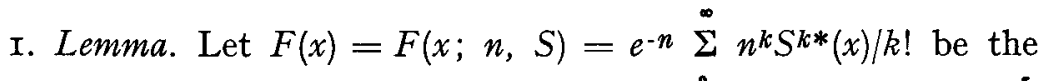
generalised Poisson function under investigation. If $S(x)=\dot{\Sigma}$ $a_{i} S_{i}(x)$, where $\Sigma a_{i}=\mathrm{I}$ (the functions $S_{i}$ need not be distribution functions, neither must the constants $a_{i}$ be real numbers of interval $[0, I])$, then

$$
F(x ; n, S)=F\left(. ; a_{1} n, S_{1}\right) * \ldots * F\left(. ; a_{r} n, S_{r}\right)(x),
$$

as is easily verified by the use of characteristic functions. This component representation is repeatedly used in the sequel.

2. A Modified Esscher Method. The Esscher method is based on an observation that the well-known Edgeworth expansion is more advantageously applicable to a conveniently modified distribution function instead of the original generalised Poisson function. Let us assume that the value of $F(x)$ is required at a point $x_{0} \geq \int_{0}^{\infty} x d F$.

1) H. Bohman and F. Esscher: Studies in Risk Theory with Numerical illustrations Concerning Distribution Functions and Stop Loss Premiums. Part I.-Skandin. Aktuarietidskrift, 3-4 (r963), pp. I72-225. 
The Esscher approximation formula is achieved by integrating over the interval $\left(x_{0}, \infty\right)$ the approximation

$$
d F(x)=e^{-n-h x-n \beta_{0}} d_{x} \Sigma c_{k} \Phi^{(k)}(z),
$$

where $\beta_{k}=\int_{0}^{\infty} x^{k} e^{h x} d S ; x_{0}=n \beta_{1} ; c_{0}=\mathrm{I} ; c_{1}=c_{2}=c_{5}=0$; $c_{3}=-\beta_{3} / 6 n^{\frac{1}{2}} \beta_{\mathbf{3}_{2}}^{2} ; c_{4}=\beta_{4} / 24 n \beta_{2}^{2} ; c_{6}=\beta_{3}^{2} / 72 n \beta_{2}^{3} ;$

$z=\left(x-n \beta_{1}\right) / \sqrt{n \beta_{2}} ; \Phi(z)=\int_{-\infty}^{z} e^{-\frac{1}{2} z^{2}} d z / \sqrt{2 \pi}$.

The approximation (I) fits best in the neighbourhood of the point $x_{0}$. By taking this observation into account it is natural to replace the equation $x_{0}=n \beta_{1}$, which defines the constant $h$, by the equation $x=n \beta_{1}$. After this modification $h$ becomes a variable, and integration gives

$$
F\left(x_{2}\right)-F\left(x_{1}\right)=\frac{e^{-n}}{\sqrt{2 \pi / n}} \int_{h_{1}}^{h_{2}} e^{-h n \beta_{1}(h)+n \beta_{0}(h)} \sqrt{\beta_{2}(h)}(\mathrm{I}+A(h)-B(h)) d h,
$$

where $\beta_{k}(h)=\int_{0}^{\infty} x^{k} e^{h x} d S ; A(h)=\beta_{4}(h) / 8 n \beta_{2}^{2}(h) ; B(h)=5 \beta_{3}^{2}(h)$ $124 n \beta_{2}^{\mathbf{3}}(h) ; x_{1}=n \beta_{1}\left(h_{1}\right) ; x_{2}=n \beta_{1}\left(h_{2}\right)$. An example, $S(x)=\mathrm{I}-e^{-x}$ is treated in the next section.

3. Exponent polynomials. By selecting $S_{i}(x)=\mathrm{I}-e^{-b_{i} x}$ in the Lemma, a component representation of $F(x)$ is gained, where each component is a modified first order Bessel function of imaginary type. If there is a device available which easily computes a strictly restricted number of convolutions, the usefulness of this method depends on how great is the required number $r$ of components, and how easily each component is computable. The calculation of one component is essentially the same problem as calculating the function

$$
F\left(x ; n, \mathbf{I}-e^{-x}\right)=e^{-n}+\int_{0}^{x} f(x) d x
$$


where

$$
f(x)=n e^{-(x+n)} \sum_{0}^{\infty} \frac{(n x)^{k}}{k !(k+\mathrm{I}) !} .
$$

For small $n$, say $\sqrt{n x}<$ ro, the series obtained by integration is easily calculated directly, but as soon as $\sqrt{n x}$ becomes great, the direct calculation is very laborious. Fortunately in this case it is possible to use the asymptotic properties of Bessel functions by means of the expansion

$$
\begin{gathered}
f(x)=\sqrt{\frac{n}{x}} \frac{e^{-(\sqrt{x}-\sqrt{n})^{2}}}{2 \sqrt{\pi} \sqrt{n x}}\left[\mathrm{I}-\frac{3}{\mathrm{I} 6}(\sqrt{n x})^{-1}-\frac{\mathrm{I} 5}{5 \mathrm{I} 2}(\sqrt{n x})^{-2}-\right. \\
\left.-\frac{\mathrm{I05}}{8 \mathrm{Ig} 2}(\sqrt{n x})^{-3}-\frac{4725}{524288}(\sqrt{n x})^{-4}-\frac{72765}{8388608}(\sqrt{n x})^{-5}\right]+ \\
\quad+\text { Remainder. }
\end{gathered}
$$

It is interesting to compare this expansion with the result obtained by the modified Esscher method for $S(x)=\mathrm{I}-e^{-x}$. On applying the formula (2) the calculation gives

$$
\frac{d F}{d x}=\sqrt{\frac{n}{x}} \frac{e^{-(\sqrt{x}-V \bar{n})^{2}}}{2 \sqrt{\pi} \sqrt{\sqrt[4]{n x}}}\left[\mathrm{I}-\frac{3}{\mathrm{I} 6}(\sqrt{n x})^{-1}\right]
$$

or the very same as the first two terms of the expansion (3). Since both the remainder of the expansion (3) and the terms of higher order, given explicitly, are insignificant unless if $\sqrt{n x}$ is quite small, the moditication (2) gives, practically speaking, an exact result in the case $S(x)=\mathrm{I}-e^{-x}$.

4. Step function approximation. If we approximate the function $S(x)$ in the Lemma by a step function, $F(x)$ becomes a multiple convolution of ordinary Poisson functions. The usefulness of this method depends on whether it is possible to rest content with a relatively small number of steps. In order to test numerically how many steps are required, it is possible to proceed as follows: Let step points of the approximation be $x_{1}<x_{2}<\ldots\left(x_{0}=0\right)$ and let

$$
\begin{gathered}
\lambda_{i}=n\left[S\left(x_{i+1}\right)-S\left(x_{i}\right)\right] \\
F_{i}(x)=e^{-\lambda_{i}} \sum_{{\stackrel{0}{m_{i}}}_{\text {appr }}}^{\left[\frac{x}{k !}\right.} \frac{\lambda_{i}^{k}}{k !} \quad\left(m_{i}=\int_{x_{i}}^{x_{i+0}} x d S /\left(S\left(x_{i+1}\right)-S\left(x_{i}\right)\right)\right.
\end{gathered}
$$


By replacing this approximation alternatively with functions $F^{-}=\Pi^{*} F_{i}^{-}$and $F^{+}=\Pi^{*} F_{i}^{+}$, where

$$
\begin{gathered}
F_{i}(x)=e^{-\frac{m_{i} \lambda_{i}}{x_{i}}} \sum_{0}^{\left[\frac{x}{x_{i}}\right]}\left\{\frac{m_{i} \lambda_{i}}{x_{i}}\right\}^{k} / k !, \text { and } \\
F_{i}^{+}(x)=e^{-\frac{m_{i} \lambda_{i}}{x_{i+1}}} \sum_{0}^{\left[\frac{x}{x_{i+1}}\right]}\left\{\frac{m_{i} \lambda_{i}}{x_{i+1}}\right\}^{k} / k !,
\end{gathered}
$$

upper and lower bounds for the approximation $F_{\text {appr }}$ are obtained in the sense that both the function $F$ and $F_{\text {appr }}$ are "approximately between" the functions $F^{-}$and $F^{+}$. All of these functions have the same mean, and the standard deviation is greatest for $F^{+}$and smallest for $F^{-}$. Owing to the steps this error estimation is not exact. A part of this question is discussed also in item 7 .

5. Monte Carlo method. The idea is to obtain by simulation a random sample $\left\{x_{1}, x_{2}, \ldots\right\}$ of a random variable, the distribution function of which is $F(x)$. For that purpose the distribution function of the number of claims

$$
P(N)=e^{-n} \sum_{0}^{N} \frac{n^{k}}{k !},
$$

is needed, and the functions

$$
S^{2^{0} *}(x)(=S(x)) ; S^{2^{1} *}(x) ; S^{2^{2} *}(x) ; \ldots ; S^{2 * *}(x)
$$

up to a sufficiently large value of $k$ (in general no more than $2^{k} \approx n+4 V(n)$. For the first simulation a random number $N$ of claims is generated by means of the distribution $P(N)$ and a random number generator. Then $N$ is written as a binary number $N=$ $\Sigma a_{k} 2^{k}\left(a_{k}=0\right.$ or I), and finally, using the formula

$$
S^{N *}=\prod_{a_{k-1}}^{*} S^{2^{k *}}
$$

the first member $x_{1}$ of the sample is obtained by means of $\Sigma a_{k}$ random numbers and of functions $S^{2^{k *}}\left(a_{k}=\mathrm{I}\right)$. By repeating the procedure sufficiently often a sample of requisite size is obtained; this sample provides directly an estimate of the function $F(x)$. 
This method is suitable in practice particularly if $n$ is small. On the other hand, if $n$ is large, difficulties arise when an attempt is made to give an accurate calculation of the tails of the highest convolutions $S^{2^{k *}}$. These problems have been treated by this author in comments at the Edinburgh meeting quoted in item I. Further notes are found below.

6. Mixed methods. By using the Lemma the function $F$ can be partitioned into several components. By applying independently to each component a proper calculation technique, different kinds of mixed methods emerge.

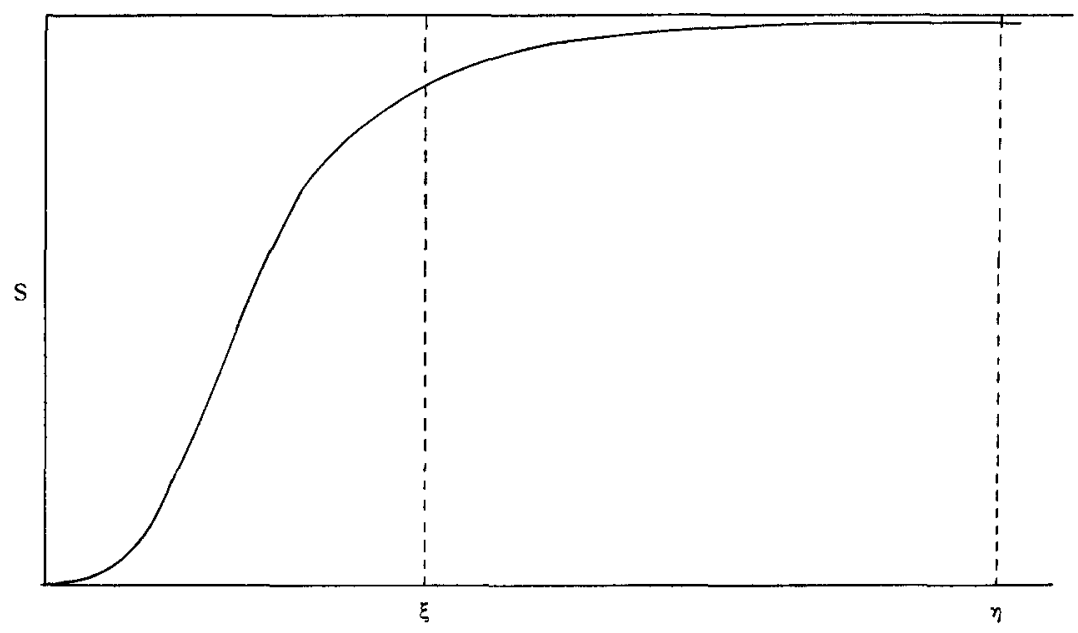

Fig. $\mathbf{I}$.

As an example a mixed method is reviewed, which Finnish insurance companies plan to use in connection with evaluation of the maximum and minimum amount of a so-called equalisation reserve. In improving this method decisive importance has been attached to the desire to obtain a single computer program which could handle all combinations of $n$ and $S$ occuring in practice. Let us assume that the function $F$ is represented in component form

$$
F=F_{1} * F_{2} * F_{3},
$$

where $F_{1}$ consists of claims $\leq \xi, F_{2}$ claims between $\xi$ and $\eta$, and $F_{3}$ of claims $>\eta$. The intention is to use a normal approximation 
for the function $F_{1}$ and the Monte Carlo method for the others. It is worthwhile to mention that in this approach it is not useful to actually calculate the functions $F_{1}, F_{2}$ and $F_{3}$ separately, since the final convolution $F_{1} * F_{2} * F_{3}$ can also be calculated by simulation simultaneously with other simulations.

The first problem is to find the greatest number $\xi$ such that $F_{1}$ can be considered to be a normal distribution. If the company is very small, then $\xi=0$ so that $F_{1}$ degenerates to the elementary distribution function $\varepsilon(x)$. If this is not the case, let

$$
F_{1}(x)=e^{-\nu} \sum_{0}^{\infty} \frac{\nu^{k}}{k !} S_{1}^{k *}(x),
$$

where $v=n S(\xi) ; S_{1}(x)=S(x) / S(\xi)$ for $x \leq \xi$. Further let $\alpha_{i}=\int_{0}^{\infty} x^{i} d S_{1}$. A necessary condition in order that $F_{1}$ can be approximated by a normal distribution is that the second term of the Edgeworth expansion is small, i.e. for all $x$

$$
\left|\frac{\mathrm{r}}{6 \sqrt{\nu}} \frac{\alpha_{3}}{\alpha_{2}^{3 / 2}} \Phi^{(3)}\left(\frac{x-\alpha_{1}}{\sqrt{\sqrt{v \alpha_{2}}}}\right)\right|<\varepsilon \text {, say, }
$$

hence

$$
\alpha_{3} / 6 \sqrt{2 \pi \nu} \alpha_{2}^{3 / 2}<\varepsilon .
$$

This condition does not indicate, strictly speaking, that for all $x$

$$
\left|F_{1}(x)-\Phi\left(\frac{x-\alpha_{1}}{\sqrt{v \alpha_{2}}}\right)\right|<\varepsilon,
$$

but for practical purposes it gives a satisfactory test especially in applications where large values of $x$ are the most important. For large values of $x$ this condition implies in general that the error is significantly less than $\varepsilon$; moreover the components $F_{2}$ and $F_{3}$ then play a decisive rôle.

This test calls for calculation of integrals $\int_{0}^{x_{i}} x^{k} d S(k=x, 2,3)$ for a sequence $x_{1}<x_{2}<\ldots$; the greatest $x_{i}$ satisfying the test inequality gives a suitable $\xi$ value.

A still faster though slightly more inaccurate, and for a small company perhaps unnecessarily severe test, is derived from the 
one described by using the following assumption, which intuitively fits practical cases in a satisfactory way. That is, if the distribution consisting of claims $\xi_{1}<x \leq \xi_{2}$ can be approximated by a normal distribution function, then the distribution consisting of claims $\leq \xi_{2}$ can also be approximated by a normal distribution. After simple calculations one obtains for $\varepsilon=$. or the following rule ${ }^{1}$ ): a suitable $\xi$ value is the greatest number satisfying the inequality

$$
S(\xi)-S\left(\frac{5}{6} \xi\right) \geq 132 / n \text {. }
$$

As soon as the number $\xi$ is found, the second limit $\eta$ can be chosen so that

$$
\frac{\mathrm{I}-S(\eta)}{\mathrm{I}-S(\xi)}=. \text { oI, say. }
$$

If $\eta$ is defined in this way, the simulation of $F_{3}$ requires in practical cases such small random numbers $N$ of claims that uncomfortably high convolutions $S_{3}^{2^{k} *}$ are not needed, and consequently no tail difficulties arise.

7. Majorant distributions. In many applications the cumbersome calculation of a generalised Poisson distribution would be avoidable if it were possible to find an easily computable distribution function more dangerous than $F(x)$. The following proposition and notably its generalisations would solve the problem in quite a satisfactory way:

$$
\begin{gathered}
\text { Proposition. Let } S(M)=\mathrm{I} \text { and } \tau=E\{X\} / M \text {, where } E\{X\}= \\
\int_{0}^{\infty} x d F \text {. Then for all } x \geq E\{X\} \\
F(x+M)>G(x),
\end{gathered}
$$

1) Let $\xi=\xi_{2}$ and $\xi t=\xi_{1}(t<\mathrm{I})$. Let $\alpha_{i}=\int_{i \xi}^{\xi} x^{i} d S /(S(\xi)-S(t \xi))$. Then $\alpha_{3} / \alpha_{2}{ }^{3 / 2} \leq t^{-3}$, so that if $v=n(S(\xi)-S(t \xi))$, then the necessary condition for normal approximation gives a rule $\nu \geq t-6 / 72 \pi \varepsilon^{2}$. In a sense an optimal value of $t$ is reached by requiring that the needed mean number of claims to length unit, $\xi_{1}$ as a measure, reaches its minimum. By differentiating the result $t=5 / 6$ is obtained. 
where

$$
G(x)=e^{-\tau} \sum_{0}^{\left[\frac{x}{M}\right]} \frac{\tau^{k}}{k !} .
$$

If also $F(x)$ is an ordinary Poisson distribution, i e.

$$
F(x)=e^{-n} \sum_{0}^{\left\lceil\frac{n x}{\tau M}\right\rfloor} \frac{n^{k}}{k !}(n \geq \tau),
$$

the proposition can be proved in the following manner:

One may assume $M=\mathrm{I}$. It has to be proved that if $x \geq \tau$ then for all $n \geq \tau$

$$
g(n)=e^{-n} \sum_{0}^{t} \frac{n^{k}}{k !}>e^{-\tau} \sum_{0}^{[x]} \frac{\tau^{k}}{k !}=G(x)\left(t=\left[\frac{n}{\tau}(x+\mathrm{I})\right]\right) .
$$

The function $g(n)$ has steps at points $n_{i}=i \frac{\tau}{x+I}$, where $i$ is an integer. Elsewhere $g^{\prime}(n)=-e^{-n_{n} t / t !}<0$. Hence, it is sufficient to prove that $g\left(n_{i}-\right)>G(x)$ for all $i>[x+\mathrm{I}]$. This requirement is certainly fulfilled if $g\left(n_{i}-\right)>g\left(n_{i-1}-\right)$ for all $i>[x+\mathrm{I}]$, since $G(x) \leq g\left(n_{[x+1]}-\right)$.

$$
\begin{aligned}
& \text { If } x \geq \tau \text {, then for all } n>\frac{[x+\mathrm{I}]}{x+\mathrm{I}} \tau\left(=n_{[x+1]}\right) \\
& \tau<[x+\mathrm{I}] \leq\left[\frac{n}{\tau}(x+\mathrm{I})\right]=t
\end{aligned}
$$

thus

$$
\begin{gathered}
n<\frac{\tau}{x+\mathrm{I}}\left(\left[\frac{n}{\tau}(x+\mathrm{I})\right]+\mathrm{r}\right)=\frac{\tau t}{x+\mathrm{I}}+\frac{\tau}{x+\mathrm{I}}<\frac{x t}{x+\mathrm{I}}+ \\
+\frac{t}{x+\mathrm{I}}=t .
\end{gathered}
$$

Consequently for all continuity points $n>n_{[x+1]}$

$$
g^{\prime \prime}(n)=e^{-n} \frac{n^{t-1}}{(t-\mathrm{I}) !}\left(\frac{n}{t}-\mathrm{I}\right)<0 .
$$


Hence for all $i>[x+\mathbf{I}]$

$$
\begin{aligned}
g\left(n_{i}-\right) & =g\left(n_{i-1}\right)+\int_{n_{i-1}}^{n_{i}} g^{\prime}(n) d n>g\left(n_{i-1}\right)+\left(n_{i}-n_{i-1}\right) g^{\prime}\left(n_{i}-\right) \\
= & g\left(n_{i-1}-\right)+e^{-n_{i-1}} \frac{n_{i-1}^{i-1}}{(i-\mathrm{I}) !}-\frac{\tau}{x+\mathrm{I}} e^{-n_{i}} \frac{n_{i}^{i-1}}{(i-\mathrm{I}) !}
\end{aligned}
$$

Since $e^{y-1} \geq y$ for all real $y$, the inequality

$$
\begin{gathered}
\frac{\tau}{x+\mathrm{I}} \leq e^{\frac{\tau}{x+1}-1}<e^{\frac{\tau}{x+1}}\left(\mathrm{I}+\frac{\mathrm{I}}{i-\mathrm{I}}\right)^{-(i-1)}=e^{n_{i}-n_{i-i}}\left(\frac{i-\mathrm{I}}{i}\right)^{i-1}= \\
=e^{n_{i}-n_{i-1}}\left(\frac{n_{i-1}}{n_{i}}\right)^{i-1}
\end{gathered}
$$

results, so that really $g\left(n_{i}-\right)>g\left(n_{i-1}-\right)$.

The author does not know whether the proposition is generally true. Intuitively it seems to be correct, but if this is the case the proof is probably not simple. 